\title{
Robust Statistical Procedures in Statistical Analysis of Controllable Noise Indicators
}

\author{
W. BATKO ${ }^{a, *}$ AND O. KNAPIK ${ }^{b}$ \\ ${ }^{a} \mathrm{AGH}$ - University of Science and Technology, Faculty of Mechanical Engineering and Robotics \\ Department of Mechanics and Vibroacoustics, al. A. Mickiewicza 30, 30-059 Krakow, Poland \\ ${ }^{b}$ Cracow University of Economics, Department of Statistics, Rakowicka 27, 31-510 Kraków
}

\begin{abstract}
The subject of the paper is statistical analysis of noise measurement results in control research of environment which is crucial for the process of identification, estimation, and interpretation of the characteristics of representative noise indicators. The proposed approach is based on robust methods of statistical analysis. In particular, the method of modeling measurement results with the use of autoregressive-moving average $(A R M A)$ time series models is described. The subjects of identification, analysis, and connected matter of verification that occur during proposed mathematical formalization of considered problem are stated. Furthermore, the question of the validity and usability of normal distribution approximation, which is very common in modeling and estimation of the expected value of the equivalent sound level, is addressed. The illustration of the proposed methodology is represented by an example of the noise analysis from the permanent monitoring in the city of Kielce. The obtained results create the background for uncertainty of estimates of calculation concerning controlled noise indicators that describe the state of acoustic environmental threats.
\end{abstract}

DOI: $10.12693 /$ APhysPolA.123.1007

PACS: 43.60.Cg, 43.50.Rq, 43.50.Yw

\section{Introduction}

The issue of risk related to evaluation of environmental acoustic threat is a subject of statutory [1-3] obligations that necessitate the implementation of statistical tests which are associated with the selection of appropriate methods of predicting acoustic risks in the analyzed field. The range of measuring and computing conditions that specify how to estimate the expected value of the controlled noise indicator, as well as the method of its uncertainty evaluation, is connected with the realization of these processes. The current estimation procedures of controlled noise indicators are derived from the classical methods of statistical inference for independent observations. The estimation [4] of their characteristics is based on the assumptions of the equivalence of the random control sample's results, on the fact that probability distribution of their occurrence's results is normal, and on the condition that the subsequent measurement observations $x_{i}, i=1, \ldots, n$ are uncorrelated with each other and therefore (in Gaussian case) independent. In the the environment acoustic threat's control, implemented on the basis of random checks, these assumptions are used without further analysis and discussion about their credibility. This applies mainly to the issue of the validity of assumption on normal distribution of noise level measurement results, as well as to the lack of correlation between them. The first one, derived from the physical properties of the measured value, is generally difficult to accept, and the other is unreliable due to the frequent appearance of

*corresponding author; e-mail: batko@agh.edu.pl interference in measurement with relatively large levels affecting the results of the subsequent sample. These concerns were a premise to undertake a statistical analysis of the results of measurements of equivalent noise level $L_{A, e q, i}, i=1, \ldots, n$, using a different method of modeling the random sample results $[5,6]$, which is crucial in the selection of the appropriate approximation and also allows an estimation of the expected value and variance of the controlled noise indicator. The aim of the study is to perform a critical analysis of the current method of the equivalent noise level estimation during verification process on the basis of classical statistical methods and propose application of robust statistical methods which promise better results. The matter of question of how reasonable is an approximation based on normal distribution assumption for the noise measurement variability for different degrees of aggregation of measurement results via calculation of the equivalent noise level is evaluated.

\section{Monitoring of acoustic threats to environment}

The program of the monitoring of acoustic threats to environment is associated with the measuring instrumentation which is used mainly during control tests. It is determined by the microphone probe, with a calibration, digital recording, and noise analysis systems (along with their software and with solutions guaranteeing the adequate transfer of archived data to the appropriate decision-making level). The machinery that was used during control tests of acoustic environmental hazards allows a direct measurement of noise level variations, i.e. the levels of $L_{A, t}[\mathrm{~dB} / \mathrm{A}], t=1,2, \ldots$ in the analyzed periods $t$. They are used to determine the base of the noise variability ratings. It is is defined by the equivalent 
level values:

$$
L_{A, e q, t}=10 \log \left[\frac{1}{M} \sum_{t=1}^{M} 10^{0.1 L_{A, t}}\right] .
$$

Their values are determined by a number of $M$ 1-second samples, adopted for the variation analysis $L_{A, e q, t}$, in selected intervals. On the basis of time series, the ratings of equivalent noise level $L_{A, e q}$, for the time: $j=1$ (6am to $6 \mathrm{pm}$ period); $j=2(6 \mathrm{pm}$ to $10 \mathrm{pm}$ period); $j=3$ (10pm to 6 am period), and the associated day-evening-night noise indicator $L_{d e n}$ are given by

$$
\begin{aligned}
& L_{\text {den }}=10 \log \left[\frac { 1 } { 2 4 } \left(12 \cdot 10^{0.1 L_{\text {day }}}\right.\right. \\
& \left.\left.\quad+4 \cdot 10^{0.1\left(L_{\text {evening }}+5\right)}+8 \cdot 10^{0.1\left(L_{\text {night }}+10\right)}\right)\right] .
\end{aligned}
$$

The yearly-average noise indicators are estimated on the basis of the following formula

$$
L_{L T}^{(j)}=10 \log \left[\frac{1}{365} \sum_{k=1}^{365} 10^{0.1 L_{A, e q, L T, k}^{(j)}}\right] .
$$

These indicators constitute underlying the choice of solutions in developed acoustic protection programs. They set the random sample $\left\{L_{\text {day }, k}, L_{\text {evening }, k}, L_{\text {night }, k}, L_{\text {den }, k}\right\}$ from a specific day of the calendar year $k=1,2, \ldots, 365$, that is used for evaluation of the acoustic risks for the environment. Their estimation is a statistical inference which is based on time-series variation $L_{L T}^{(j)}, L_{L T, t}, t=1,2, \ldots, n$ created from the results of "random-snapshot" of acoustic environmental studies. Control tasks defined this way is a perfect area to use robust statistical methods. The proposed methods were not used in the previous studies of acoustic environmental hazards. The purpose of this paper is to recommend them through presentation of the research work carried out. Motivation for use of such methods is based on the fact that a number of unusual acoustic interferences (definitely different from the others, which can be seen as outliers) during the control process are present. Thus, statistical analysis of this type of time series, conducted using methods derived from classical statistics, may not provide valid results. The condition above may indicate the desirability of a broader introduction of methods derived from robust statistics to environmental research practices.

\section{3. $A R M A$ models in the analysis of noise indicators}

Following the approach of papers [5-7] in the process of acoustic control data modeling, we assume in this work that the probabilistic model for the present measurements is a stochastic process, that is a sequence of random variables. Time series representing the results of the next "snapshot" control observations $x_{1}, x_{2}, \ldots, x_{n}$, describes environment's acoustic condition. It is assumed that the probabilistic structure of the change of control results can be represented via the following mechanism:

$$
\begin{aligned}
& \left(x_{t}-\mu\right)-\phi_{1}\left(x_{t-1}-\mu\right)-\ldots-\phi_{p}\left(x_{t-p}-\mu\right) \\
& \quad=u_{t}+\theta_{1} u_{t-1}+\ldots+\theta_{q} u_{t-q},
\end{aligned}
$$

where $\left\{u_{t}\right\} \sim W N\left(0, \sigma_{u}^{2}\right)$ which is white noise with mean 0 and variance $\sigma_{u}^{2}$. The process $\left\{x_{t}, t \in Z\right\}$ is called $\operatorname{ARMA}(p, q)$.

The attention is directed to stationary time series models which can be well approximated by the use of $A R M A(p, q)$ models. In the classical approach to identification and estimation of $A R M A$ processes, the method of construction based on likelihood function for the considered models and methods based on a comparison of the estimated models using information criteria (i.e. Akaike, Schwarz) are used predominately. However, they require additional assumptions about the distribution of the random error. Generally, it is assumed that it is a zero-mean Gaussian white noise. This assumption will be adopted throughout this paper. The selection of the appropriate $A R M A(p, q)$ process model is connected with the choice of order of $q$ corresponding to the moving average part of the process, and $p$ corresponding to autoregressive part of the process. It is usually made on the basis of the value of information criterion, e.g. Akaike. Technical details of classical estimation procedure can be found e.g. in [8] or [9]. The last step of this procedure is to check whether the model residuals follow the normal distribution. There are many types of tests for normality. In this paper, in the empirical studies, the Jarque-Bery test for normality (see [10]) was used. It is based on two characteristics of empirical distributions, that is the skewness and the kurtosis, which are compared with theoretical parameters of the normal distribution.

One of the possible ways of robust estimating of parameters of ARMA's models is known as $F \tau$-estimation, introduced by [11-13]. The description of the procedure will follow these references. According to them, it is assumed that we observe realization of the following process

$$
y_{t}=x_{t}+v_{t}, \quad t=1, \ldots, T,
$$

where $v_{t}$ is a outlyingness process and $x_{t}$ is $A R M A(p, q)$ process, where random variables $u_{1}, \ldots, u_{T}$ are independent and normally distributed $N\left(0, \sigma_{u}^{2}\right)$.

Let $\hat{u}_{t}(\lambda)$ be the residuals from the considered model calculated with the use of Kalman filter and $\Sigma_{t}^{2}(\lambda)=$ $a_{t}^{2} \sigma_{u}^{2}$ is variance of $\hat{u}_{t}$. Method of maximum likelihood estimation (MLE) is obtained by maximizing the reduced likelihood function, the logarithm of which equals, up to constant, the following equation:

$$
\begin{aligned}
& Q(\lambda)=\sum_{t=1}^{T} \log \left(a_{t}^{2}(\lambda)\right) \\
& +T \cdot \log \left(\frac{1}{T} \sum_{t=1}^{T}{\frac{\hat{u}_{t}^{2}(\lambda)^{2}}{a} t}_{t}(\lambda)\right) .
\end{aligned}
$$

It is well known that the MLE estimator is very sensitive to presence of outliers. To make MLE estimator more robust, we can replace $Q(\lambda)$ by 


$$
\begin{aligned}
& Q_{\tau}(\lambda)=\sum_{t=1}^{T} \log \left(a_{t}^{2}(\lambda)\right) \\
& \quad+T \cdot \log \left(s^{2}\left(\frac{\hat{u}_{1}(\lambda)}{a_{1}(\lambda)}, \ldots, \frac{\hat{u}_{T}(\lambda)}{a_{T}(\lambda)}\right)\right),
\end{aligned}
$$

where $s$ is a $\tau$ scale parameter estimator defined in [14].

In order to improve the properties of the estimator, Bianco and others (see [11]) proposed to replace the residuals $\hat{u}_{t}$ with the filtered residuals $\tilde{u}_{t}$ based on the procedure of the modification of a state space representation of the process. Technical details of this method can be found in [11]. The estimator $\lambda=\max _{\lambda}\left(Q_{\tau}^{*}(\lambda)\right)$, where

$$
\begin{aligned}
& Q_{\tau}^{*}(\lambda)=\sum_{t=1}^{T} \log \left(\tilde{a}_{t}^{2}(\lambda)\right) \\
& \quad+T \cdot \log \left(s^{2}\left(\frac{\tilde{u}_{1}(\lambda)}{\tilde{a}_{1}(\lambda)}, \ldots, \frac{\tilde{u}_{T}(\lambda)}{\tilde{a}_{T}(\lambda)}\right)\right)
\end{aligned}
$$

is called the estimator of the method of filtered $\tau$ estimation, $F \tau$ estimator for short.

The procedure above can be completed using robust test of the normality of residuals. It is well-known that the classic Jarque-Bera ([10]) normality test is, as based on the central moments of the empirical distribution of data, non-robust. In the literature there are several proposals to modify the Jarque-Bera normality test in order to make it more robust (see $[15,16]$ ). G. Brys, M. Huber and A. Struyf [15] suggest building a normality test (Jarque-Bera type) based on the robust measure of asymmetry, called medcouple $(M C)$ and related to distribution tail measures such as the left medcouple $(L M C)$ and the right medcouple $(R M C)$. These measures can be defined as follows:

$$
M C_{n}=\underset{x_{1} \leq m_{F} \leq x_{2}}{\operatorname{med}} h\left(x_{1}, x_{2}\right),
$$

where $h\left(x_{i}, x_{j}\right)=\frac{\left(x_{j}-m_{F}\right)-\left(m_{F}-x_{i}\right)}{x_{j}-x_{i}}, m_{F}=F_{n}^{-1}(0.5)$, $L M C_{n}=-M C_{n}\left(x<m_{F}\right), R M C_{n}=M C_{n}\left(x>m_{F}\right)$. Null and alternative hypothesis are the same as in the Jarque-Bera normality test. Test statistic takes, under $H_{0}$, the following form

$$
T=n(w-\omega)^{T} \Sigma_{k}^{-1}(w-\omega) \approx \chi^{2}(k),
$$

where $w=\left(M C_{n} L M C_{n} R M C_{n}\right), \omega=\left(\begin{array}{lll}0 & 0.199 & 0.199\end{array}\right)$, and

$$
\Sigma_{3}=\left(\begin{array}{ccc}
1.25 & 0.323 & -0.323 \\
0.323 & 2.62 & -0.0123 \\
-0.323 & -0.0123 & 2.62
\end{array}\right) .
$$

This procedure ends statistical analysis of time series. In the next section of the article, ideas of robust statistical analysis of environmental noise control results are presented.

\section{Empirical example}

The results of continuous noise monitoring measurements, made in the town of Kielce are an exemplification of the proposed statistical analysis. The data is contained in the Department of Mechanics and Vibroacoustics database of acoustic monitoring system (www . monitoringakustyczny.pl). The base of the performed analysis were 3 time series. Ont of them represented the original course of the noise level measurements $L_{A, i}$, recorded at 1 second intervals, and two others were representative of aggregated time series created from the value of the equivalent sound level $L_{A, e q, i}$ in 1 and 5 -minute intervals.

The use of classical statistical procedures for identification and estimation of the parameters of the time series shows that the original data (the sound level series measured every second) has the strongest dependency structure. The best model within the $A R I M A$ class according to the AIC criterion for that data is the $A R I M A(3,1,5)$ model. The estimates of $A R I M A$ process obtained on the basis of the classical statistical methods were also obtained on the basis of robust estimation procedure. The identification procedure has found 792 outliers in the data. Moreover, it is worth mentioning that classical statistical analysis indicates that the examined series is nonstationary (this requires a formal testing) and the series needs to be differentiate in order to make it stationary. The reason for this may be also the presence of outliers in the data. The results of applying the two estimation procedures are summarized in Table I.

TABLE I

Results of estimation of $A R M A$ models for original data.

\begin{tabular}{c|c|c|c|c|c|c|c|c}
\hline \hline Method & $A R(1)$ & $A R(2)$ & $A R(3)$ & $M A(1)$ & $M A(2)$ & $M A(3)$ & $M A(4)$ & $M A(5)$ \\
\hline$M L E^{*}$ & 0.7273 & 0.4798 & -0.2947 & -0.3668 & -0.5475 & 0.0546 & -0.0691 & -0.0688 \\
\hline$F \tau$ & 0.3278 & 0.0442 & -0.1272 & -0.2578 & -0.1238 & -0.1626 & -0.0538 & -0.0195 \\
& $(0.1328)$ & $(0.3725)$ & $(0.3346)$ & $(0.0315)$ & $(0.1872)$ & $(0.3345)$ & $(0.0056)$ & $(0.0055)$ \\
\hline
\end{tabular}

${ }^{*}$ Due to numerical problems the standard errors were not obtained.

There are two crucial issues in the analysis carried out. Firstly, ARIMA model parameter estimates differ significantly, depending on the approach used, classical or robust. Secondly, the obtained robust estimates of 
$A R I M A$ parameters are mainly not statistically significant which may suggest that the model considered in accordance with the classic AIC criterion for the best model is totally inadequate to the data. The approximation of the distribution based on the normal distribution is also not relevant. As we are dealing with dependent data, calculation of classical characteristics such as the sample mean, the sample variance, as well as the kernel estimation of density function, determination of entropy, etc., is not justified.

The next step in the analyzes was to determine the 60 -second averages and to create the $L_{A, e q}$ average series - noise indicators, that is the series of equivalent noise level in every minute. The classical statistical procedure of finding the best $A R I M A$ model for the data shows the $\operatorname{ARIMA}(4,1,2)$ model. The dependency between the observations exists, but is slightly weaker than in the original series. The procedure continues to indicate the need to calculate the first differences in order to bring the series to stationary case. In this case, a robust estimation method of model parameters was also used. Observations that can be considered as outliers were identified. The procedure of identifying outliers indicated the presence of four such values. The results are contained in Table II.

TABLE II

Results of estimation of $A R M A$ models for 1-minute equivalent noise level indicators.

\begin{tabular}{c|c|c|c|c|c|c}
\hline \hline Meth. & $A R(1)$ & $A R(2)$ & $A R(3)$ & $A R(4)$ & $M A(1)$ & $M A(2)$ \\
\hline MLE & -0.6734 & -0.1184 & 0.0797 & -0.1337 & -0.4887 & -0.4793 \\
& $(0.1007)$ & $(0.0512)$ & $(0.0474)$ & $(0.0457)$ & $(0.0966)$ & $(0.0955)$ \\
\hline$F \tau$ & -0.6400 & -0.0272 & 0.1555 & -0.0989 & 0.4997 & 0.4989 \\
& $(0.0426)$ & $(0.0890)$ & $(0.0487)$ & $(0.0412)$ & $(0.0798)$ & $(0.0800)$
\end{tabular}

Approximation of the distribution of the indicators with a normal distribution is also not possible in this case.

Finally, time series of five minute equivalent noise levels was analyzed. A statistical procedure based on classical methods suggests that best $A R I M A$ model is in this case $A R I M A(0,0,0)$ with the average level equaling 67 . On the basis of robust estimation procedure similar estimates of the parameters of the model were obtained. The identification procedure reveals presence of 3 outliers in the data. The normality was tested with a robust test in order to determine whether the considered time series can be considered a Gaussian white noise. The results are contained in Table III.

The result of robust test of normality (Jarque-Bera type, as proposed in [15]), p-value of 0.5976585 , ultimately confirms white noise type and Gaussian nature of the considered time series. However, the estimation process of the parameters of the process, due to presence of outliers, should be based on robust statistical methods. In this case, it is justified to use statistical methods for independent observations, such as calculating the sam-

\section{TABLE III}

Results of estimation of $A R M A$ models for 5 -minutes equivalent sound level indicators.

\begin{tabular}{c|c}
\hline \hline Method & Intercept \\
\hline$M L E$ & $67.5582(0.0921)$ \\
$F \tau$ & $67.4789\left(^{*}\right)$ \\
\hline
\end{tabular}

*Due to numerical problems the standard errors were not obtained.

ple mean, the sample variance, the kernel estimation of density function etc.

\section{Conclusions}

Statistical formalization for noise measurements' modeling in one of the busiest street of Kielce presented in the paper indicated the desirability of using the robust statistics methods. Identification of serious disturbances in the mechanism creating the acoustic climate within the analyzed area - which have a great impact on the estimation process of controlled noise indicators - was proposed. In particular, the process of creating the results of control tests' modeling mechanism was analyzed. The need for the analysis of Gaussian nature of controlled indicators was investigated.

In the paper it was shown to what errors could the use of classical methods of statistical analysis of time series lead, especially in the situation of presence of outliers caused by random environmental disturbances in the data. The results obtained in this paper led to discovery of very important aspects of analysis connected with the proper approximation of models that describe the mechanism of noise indicators controlled value changes which secures the conditions of the expected value estimations and the variance of long-term noise indicators. The following conclusions can be stated:

- From perspective of the authorities that control the acoustic state of the environment, it is preferred to conduct the analysis of results to time series created on the basis of two or five minutes equivalent noise level $L_{A, e q}$ time series. For such time series, statistical methods for independent observations work well. The equivalent noise level $L_{A, e q}$ series posses the character of the Gaussian white noise. However, for the purpose of estimation of unknown parameters of the distribution it is better to use robust statistical methods.

- From the forecasting point of view, time series of one-minute equivalent sound level would be a much better choice. However, the analysis of this type of series requires the use of methods derived from the time series analysis (dependent observations) and robust statistics. The classical statistical procedure of analysis of monitoring data in the form of time 
series equivalent noise level can lead to serious errors. Using robust methods is a better alternative.

- In the process of statistical inference, significant attention is to be paid to the process of data aggregation via calculation of equivalent noise level time series. There is a general tendency associated with data aggregation, namely, with the transition to the more aggregate time series by averaging $\left(L_{A, e q, T}\right)$ we get simpler models. Dependencies between observations are becoming "weaker".

Results obtained from the study are important indications for the process aimed to select an appropriate model approximation, describing the mechanism of subsequent ratings control variations. They form the basis for further considerations that can go in the following directions:

- Robust analysis of time series of different noise indicators used directly by the regulatory authorities (long-term indicators).

- The development and implementation of robust methods for identification of a time series model.

\section{References}

[1] Directive 2002/49/WE of the European Parliament and of the Council of 25 June 2002, relating to the assessment and management of environmental noise, Official Journal of the European Communities, 18 July 2002.

[2] The Act of 27-th April 2001, Environment Protection Law, J. Law 2008 No. 25, item 150 with later amendments).

[3] Regulation of the Minister of Environment, of 2-nd October 2007, concerning requirements of performing in the environment the measurements of substances or energy levels for managers of roads, railways, tramways, airports and harbors J. Law 2007, No. 192, item 1392.
[4] Guide to the Expression of Uncertainty Measurement. International Organization for Standardization, ISBN 92-67-10188-9, 1995.

[5] W. Batko, R. Bal-Pyrcz, Archiv. Acoust. 31, 253 (2006).

[6] R. Bal, Ph.D. Thesis, WIMiR AGH University of Science and Technology, Kraków 2011.

[7] W. Batko, R. Bal, Archiv. Acoust. 35, 361 (2010).

[8] G.E.P. Box, G.M. Jenkins, G.C. Reinsel, Time Series Analysis: Forecasting and Control, Wiley Series in Probability and Statistics, 4 edition, Wiley, New Jersey 2008.

[9] P.J. Brockwell, R.A. Davis, Introduction to Time Series and Forecasting, 2nd edition, Springer, New York 2002.

[10] A.K. Bera, M. Jarque, Internat. Statist. Rev. 55, 163 (1987).

[11] A.M. Bianco, B.M. Garcia, E.J. Martinez, V.J. Yohai, COMPSTAT 96, Proceedings in Computational Statistics, Ed. A. Prat, Physica-Verlag, Heidelberg 1996, p. 27.

[12] A.M. Bianco, B.M. Garcia, E.J. Martinez, V.J. Yohai, J. Forecast. 20, 565 (2001).

[13] R.A. Maronna, D.R. Martin, V.J. Yohai, Robust Statistics: Theory and Methods, John Wiley and Sons, New York 2006.

[14] R.A. Yohai, R.H. Zamar, JASA, 86, 403 (1988).

[15] G. Brys, M. Hubert, A. Struyf, Symposium A Quarterly Journal In Modern Foreign Literatures, PhysicaVerlag, Heidelberg 2004, p. 753.

[16] Y.R Gel, W. Miao, J.L. Gastwirth, Comp. Stat. Data Analys. 51, 2734 (2007). 